\title{
Immunophenotype of Erythroid Precursors in Patient with Pure Red Cell Aplasia (PRCA): Utility of Analysis of Erythroid Maturation
}

$\mathrm{J}$ erez $\mathrm{J}^{1 *}$ and Ocqueteau $\mathrm{M}^{2}$

${ }^{1}$ Department of Internal Medicine, Pontifical Catholic University of Chile, Chile

${ }^{2}$ Department of Hematology and Oncology, Pontifical Catholic University of Chile, Chile

*Corresponding author: J oaquín J erez, Department of Internal Medicine, Pontifical Catholic University of Chile, School of Medicine, Marcoleta 367, Santiago, RM, Chile

Received: March 16, 2021; Accepted: May 03, 2021;

Published: May 10, 2021

\section{Introduction}

Pure Red Cell Aplasia (PRCA) is an infrequent disease [1,2], which usually presents as hypogenerative normochromic anemia, and is characterized by a significant decrease (including absence) of erythroid precursors [3]. Its etiology can be congenital or acquired, and its correct diagnosis requires exclusion of alternative cases of refractory anemia, so the bone marrow histology plays a crucial role. Myelodisplastic Syndromes (MDS) should always be considered in its differential diagnosis. The use of laboratory tools, specifically Flow Cytometry (FCM) is gained importance in the study of malignant and benign hematology pathologies. In MDS, FCM is not yet considered a standard of care, however it provides valuable information $[4,5]$ and there are numerous publications and scores for its usual clinical use (for example Ogata score and RED-score [6,7]). In relation to the rise of FCM in MDS, enormous progress has been made in the description of the erythroid precursors immunophenotype [8-10].

An example of normal erythroid maturation is presented in Figure 1, showing proerythroblasts with immunophenotype $\mathrm{CD} 71^{+}$ $\mathrm{CD}_{105^{+}} \mathrm{CD} 117^{+}$, basophilic erythroblasts $\mathrm{CD} 71^{+} \mathrm{CD} 105^{+} \mathrm{CD} 117^{-}$, polychromatophilic and orthochromatophilic erythroblasts $\mathrm{CD} 71^{+}$ CD105 CD117 distinguishing by size in Forward Scatter (FSC) versus CD36 respectively. Characteristic maturation curve in CD117 versus CD105 analysis evidenced a predominance towards more mature erythroblasts.

\section{Methodology}

\section{Study design and patient selection}

An observational, retrospective and descriptive study was carried out. Adult patients between 2008 and 2018 with PRCA were included, a diagnosis that comprehended a bone marrow biopsy with a concordant study with FCM in said sample. The FCM study was carried out with marking tubes for myelodysplasia according to the Euroflow 2012 consensus [11], which meant that patients studied prior to 2012 did not have erythroid maturation information with CD71 and CD105 markers. Patients with aplastic anemia and patients with maturational disorders in other hematopoietic lines were excluded.

The information was obtained from the database of the Cancer Center of the UC-Christus Health Network, Department of Hematology and Oncology and by reviewing the Electronic Clinical Record. Prior to data analysis, the identity of the patients was omitted, as recommended by the Scientific Ethics Committee.

\section{Objectives and variables studied}

The main objective was to analyze the cytometric variables in patients with a confirmed diagnosis of PRCA. Specifically, variables regarding erythroblast percentage, defined as CD71+CD45, percentage of $\mathrm{CD} 117^{+}$and $\mathrm{CD} 105^{+}$erythroblasts, Coefficients of Variation (CV) of CD36 and CD71 were analyzed. As secondary variables, the percentage of precursors (divided into lymphoid and myeloid) and the percentage of $\mathrm{T}$ and B lymphocytes, respectively, were analyzed. In turn, the ratio MFI CD45 erythroblasts/MFI CD45 lymphocytes was devised as a parameter of deviation towards more mature lines (the lower the value, the greater the deviation). As a secondary objective, a comparison was made in the cytometric variables between patients with PRCA and patients with myelodysplasia obtained from our database between years 2013-2018, with a percentage of erythroblasts $<10 \%$ total (where there could be diagnostic doubt). The presence of significant contamination of the samples analyzed with peripheral blood was ruled out, verifying the presence of mast cells with CD117 marker.

Differences were estimated using the log-rank or Gehan-BreslowWilcoxon method as appropriate. The analysis was performed with GraphPad Prism software. A value of $\mathrm{p}<0.05$ was considered significant.

\section{Results}

Six patients with PRCA were reported, the average age was 52 years and there was the same proportion according to sex (1:1). 50\% had some secondary cause: systemic lupus erythematosus, parvovirus infection in a patient with HIV, and thymoma were reported. The average hemoglobin at the time of diagnosis was $6.05 \mathrm{gr} / \mathrm{dL}$, with a reticulocyte index of $<1 \%$ in all cases.

Regarding cytometric parameters, it is noteworthy that only two cases were presented after 2012, so the maturational analysis of the erythroid line could only be carried out on these samples. In both cases (Figure 2), the absence of $\mathrm{CD}_{1} 17^{+}$erythroblasts practically stand out, as do $\mathrm{CD} 105^{+}$erythroblasts; with a ripening curve clearly deviated towards the more mature line. Regarding the CVs of CD36 and CD71, both were less than $65 \%$ and $80 \%$ respectively (cut-off points suggested by RED-score [7], higher values suggest MDS). 

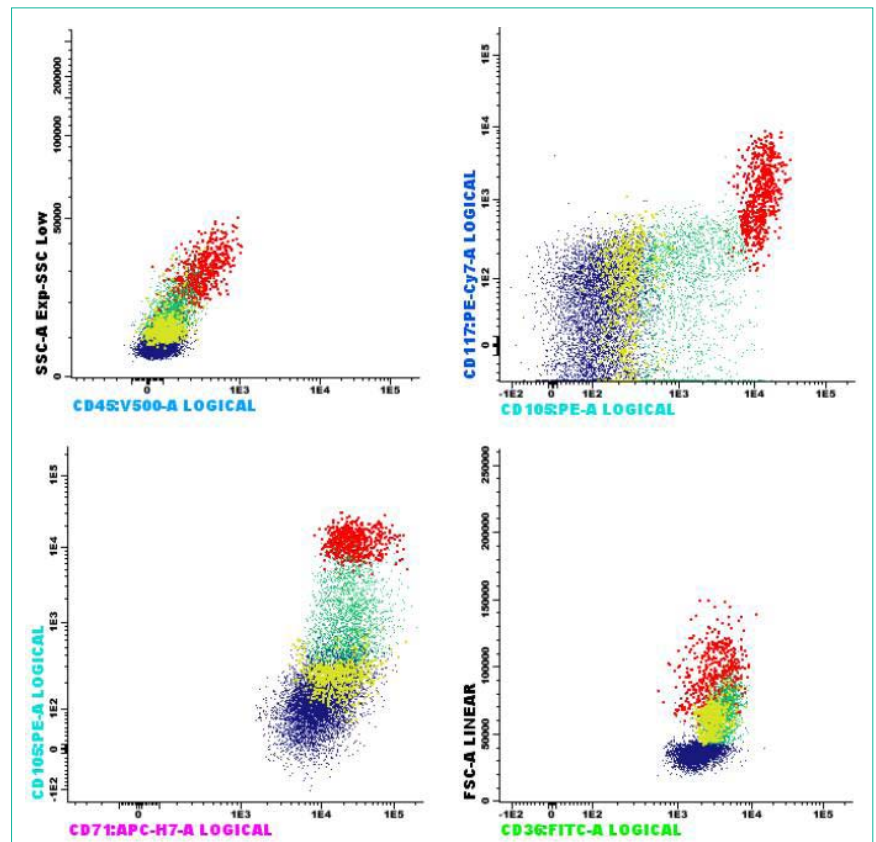

Figure 1: An example of normal erythroid maturation by flow cytometry.
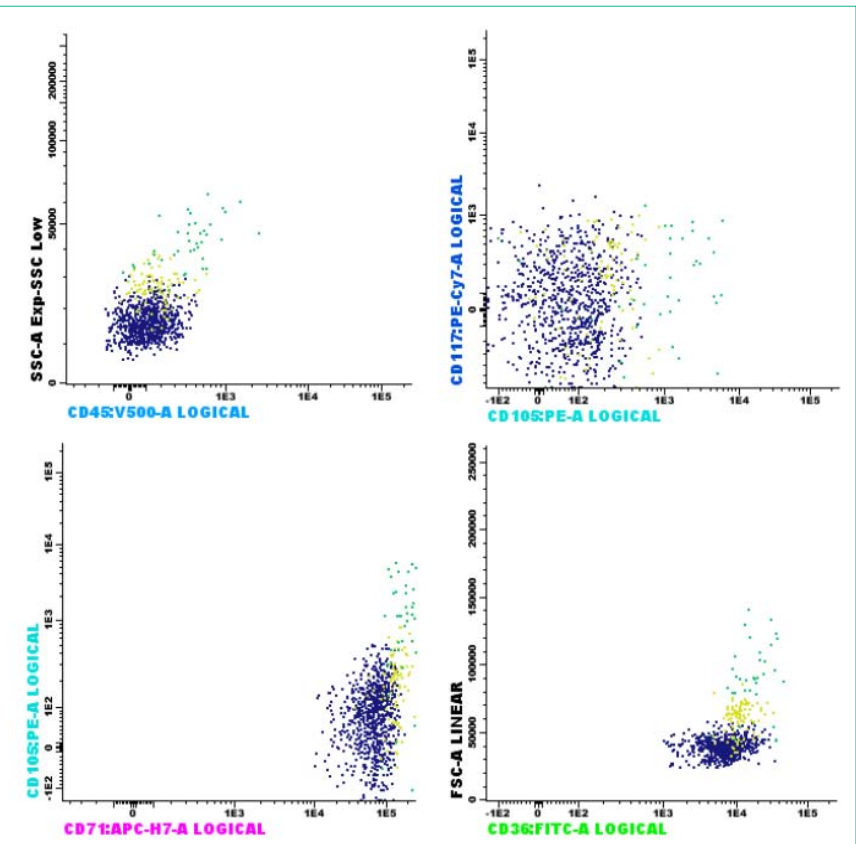

Figure 2: Typical pattern of PRCA by flow cytometry, characteristically with absence of erythroblast $\mathrm{CD} 117^{+}$and $\mathrm{CD} 105^{+}$.

Regarding the rest of the parameters analyzed (Table 1), it should be noted that all of the patients had $<1 \% \mathrm{CD} 34^{+}$precursors, all had erythroblast count $<1 \%$ of the total. The mean ratio of MFI CD45 erythroblast/MFI CD45 lymphocyte was 0.01 .

Twelve cases of MDS with erythroblasts $<10 \%$ were reported, with an average age of 73 years and mostly women (58\%). When comparing the immunophenotype of patients with PRCA with patients with MDS (Table 1), it immediately stands out that only one patient presented a percentage of erythroblasts $<1 \%$ and with a
Table 1: Cytometric variables of patients with PRCA and MDS with low count of erytroblasts.

\begin{tabular}{|c|c|c|c|}
\hline & PRCA & MDS & \\
\hline $\begin{array}{l}\text { Precursors CD34+ } \\
\text { (\%total. range) }\end{array}$ & $0.41(0.28-0.70)$ & $2.15(0.03-5)$ & $p<0.05$ \\
\hline $\begin{array}{l}\text { a) Myeloid precursors } \\
\text { (\%relative) }\end{array}$ & $86.01(67.04-93.44)$ & 99.33 (96.89-100) & $\mathrm{p}<0.05$ \\
\hline $\begin{array}{l}\text { b) Lymphoid precursors } \\
\text { (\%relative) }\end{array}$ & $13.99(6.36-32.3)$ & $0.66(0-3.11)$ & $p<0.05$ \\
\hline Erythroblasts (\%total)* & $0.46(0.12-0.92)$ & $4.42(0.3-0.9)$ & $p<0.05$ \\
\hline $\begin{array}{l}\text { a) Erythroblasts CD117 } \\
\text { (\%relative) }\end{array}$ & 0 & $9.43(1.14-30.92)$ & \\
\hline $\begin{array}{l}\text { b) Erythroblasts CD117 } \\
\text { CD105 (\%relative) }\end{array}$ & $0.01(0-0.02)$ & $14.69(5.31-30)$ & \\
\hline c) CV CD36 & $54.75(41-68.5)$ & $75.93(51.6-132)$ & \\
\hline d) CV CD71 & $40.6(31-50.2)$ & $55.13(38.8-77)$ & \\
\hline Lymphocytes (\%total) & $19.31(7.18-39)$ & $14.77(2-33)$ & $p=0.29$ \\
\hline $\begin{array}{l}\text { a) T-Lymphocytes } \\
\text { (\%relative) }\end{array}$ & $16.80(6.96-38)$ & $12.91(1.5-31)$ & $p=0.36$ \\
\hline MFI CD45 Erythroblasts/ & \multirow{2}{*}{$0.01(0.003-0.044)$} & \multirow{2}{*}{$0.03(0.01-0.07)$} & \multirow{2}{*}{$p=0.28$} \\
\hline Lymphocytes & & & \\
\hline
\end{tabular}

clear presence of erythroblasts $\mathrm{CD} 117^{+}$and $\mathrm{CD} 105^{+}$. Regarding the precursor compartment, there was a significant difference with higher values in patients with MDS (in accordance with what was reported by the Ogata score), and a significant decrease in the lymphoid precursor compartment. Both the MFI CD45 ratio and the CVs were not useful as discriminators. The ripening curves scarcely showed alteration, with little predominance towards more immature lines.

\section{Discussion}

PRCA is an infrequent entity, whose presentation is nonspecific and its main differential diagnosis by prevalence would be MDS. The present report is the first in the literature to analyze the erythroblast immunophenotype in patients with PRCA. There are previous reports in pathologies with a similar pathophysiology such as aplastic anemia $[12,13]$, however, the number of $\mathrm{CD} 34^{+}$precursors and the inverted CD4/CD8 lymphocyte ratio are described as significant parameters [14]. The present study shows that the immunophenotype coincides with those described in the pathophysiology: the most immature precursors in the erythroid series are affected, portraying that the erythroid maturation arrest in $78 \%$ of the cases would be prior to the proerythroblasts.

In contrast to patients with MDS, where it can be observed an increase in CD34 with a tendency in erythroid maturation towards more immature lines, but without frank maturational arrest. In our clinical experience, it was rare to find a gross alteration in erythroid maturation in patients with MDS, unlike our patients with PRCA. The same is corroborated in a recent study [8], in which the percentage of $\mathrm{CD} 117^{+}$and $\mathrm{CD} 105^{+}$erythroblasts in the multivariate analysis is not associated with an increased risk of MDS. It is striking in the present study that CVs in MDS do not achieve the suggested values, an element that can be explained by the selection of patients with decreased erythroblasts.

The main disadvantage of this report is the low number of patients, so it is necessary to verify these findings in a series with a greater number of patients. It remains uncertain the evaluation of other parameters that include subtypes of T lymphocytes (such as the 
$\mathrm{CD} 4 / \mathrm{CD} 8$ ratio). And the concern arises about being able to generate parameters that are predictive of response to therapy, such as the percentage of $\mathrm{CD} 117^{+}$and $\mathrm{CD}_{105^{+}}$erythroblasts at diagnosis.

In conclusion, in patients with PRCA, analysis in maturation by FCM could be useful to guide diagnosis and holistic hematopoietic analysis focused mainly on $\mathrm{CD}^{+} 4^{+}$precursor levels may help to differentiate from MDS with relative decrease in erythroid series.

\section{References}

1. Fisch $\mathrm{P}$, Handgretinger $\mathrm{R}$, Schaefer $\mathrm{H}$. Pure red cell aplasia. $\mathrm{Br} \mathrm{J}$ Haematol 2000; 111: 1010-1022.

2. Malhotra $P$. Spectrum of pure red cell aplasia in adult population of north-west India. Hematology. 2008; 13: 88-91.

3. Means RT. Pure red cell aplasia. Blood. 2016; 128: 2504-2509.

4. Cameirao B. The Use of Flow Cytometry in Myelodysplastic Syndromes: A Review. Front Oncol. 2017; 7: 270.

5. Aanei CM. Diagnostic Utility of Flow Cytometry in Myelodysplastic Syndromes. Front Oncol. 2016; 6: 161.

6. Ogata K. Diagnostic application of flow cytometric characteristics of CD34 cells in low-grade myelodysplastic syndromes. Blood. 2006; 108:1037-1044.

7. Mathis S. Flow cytometric detection of dyserythropoiesis: a sensitive and powerful diagnostic tool for myelodysplastic syndromes. Leukemia. 2013; 27 : 1981-1987.
8. Westers TM. Immunophenotypic analysis of erythroid dysplasia in myelodysplastic syndromes. A report from the IMDSFlow working group. Haematologica. 2017; 102: 308-319.

9. Fajtova M. Immunophenotypic profile of nucleated erythroid progenitors during maturation in regenerating bone marrow. Leuk Lymphoma. 2013; 54: 2523-2530.

10. An X. Flow Cytometry (FCM) Analysis and Fluorescence-Activated Cell Sorting (FACS) of Erythroid Cells. Methods Mol Biol. 2018; 1698: 153-174.

11. van Dongen JJ. EuroFlow antibody panels for standardized n-dimensional flow cytometric immunophenotyping of normal, reactive and malignant leukocytes. Leukemia. 2012; 26: 1908-1975.

12. Otawa M. Comparative multi-color flow cytometric analysis of cell surface antigens in bone marrow hematopoietic progenitors between refractory anemia and aplastic anemia. Leuk Res. 2000; 24: 359-366.

13. Huang M. Immunophenotype of myeloid granulocytes: a pilot study for distinguishing myelodysplastic syndrome and aplastic anemia by flow cytometry. Int J Lab Hematol. 2010; 32: 275-281.

14. Mentzel $U$. Analysis of lymphocyte subsets in patients with aplastic anemia before and during immunosuppressive therapy. Ann Hematol. 1993; 66: $127-$ 129. 